\title{
AVALIAÇÃO DE FUNÇÕES DE AFILAMENTO PARA A ESTIMATIVA DE DIÂMETRO DE ESPÉCIES FLORESTAIS COMERCIAIS DO BIOMA AMAZÔNICO MATO-GROSSENSE
}

\author{
Luciano Rodrigo Lanssanova ${ }^{1}$, Jaime Antônio Ubialli ${ }^{2}$, Julio Eduardo Arce ${ }^{3}$, Allan Libanio Pelissari ${ }^{4}$, \\ Cyro Matheus Cometti Favalessa ${ }^{1}$, Ronaldo Drescher ${ }^{2}$ \\ ${ }^{1}$ Eng. Florestal, M.Sc., UFMT, Cuiabá, MT, Brasil - lanssanova@ florestal.eng.br; cyromatheus@hotmail.com \\ ${ }^{2}$ Eng. Florestal, Dr., UFMT, Cuiabá, MT, Brasil - jubialli@yahoo.com.br; ronaldodrescher@ gmail.com \\ ${ }^{3}$ Eng. Florestal, Dr., Depto. de Ciências Florestais, UFPR, Curitiba, PR, Brasil - jarce@ufpr.br \\ ${ }^{4}$ Eng. Florestal, Doutorando em Eng. Florestal, UFPR, Curitiba, PR, Brasil - allanpelissari@gmail.com
}

Recebido para publicação: 10/04/2012 - Aceito para publicação: 25/03/2013

\begin{abstract}
Resumo
O objetivo do presente trabalho foi ajustar, avaliar e selecionar funções de afilamento para a estimativa de diâmetros ao longo do fuste para espécies florestais comercialmente exploradas na Amazônia Mato-grossense. O estudo foi desenvolvido em unidades de manejo florestal, em áreas de Floresta Amazônica, localizadas no município de Santa Carmem, estado do Mato Grosso. Para o ajuste das funções de afilamento, foram cubadas 449 árvores das espécies florestais Goupia glabra, Qualea albiflora, Trattinnickia burseraefolia, Erisma uncinatam e Mezilaurus itauba. Foram ajustados os modelos não segmentados de Schöepfer (1966), Kozak et al. (1969), Hradetzky (1976) e Demaerschalk (1972) adaptado por Moura (1994) e o modelo segmentado de Max e Burkhart (1976). Para avaliar a acuracidade dos ajustes, foi utilizado o coeficiente de determinação ajustado, o erro padrão de estimativa em porcentagem e a análise gráfica dos resíduos, além do valor ponderado dos escores estatísticos e do cálculo das estatísticas auxiliares: desvio, desvio padrão das diferenças, somatório dos quadrados dos resíduos relativos e resíduos percentuais. A função de afilamento de Demaerschalk (1972) adaptada foi a mais acurada para estimar os diâmetros ao longo do fuste de espécies florestais da Amazônia Mato-grossense. Essa função permite descrever a forma dos troncos e possibilita a determinação de sortimentos para múltiplos produtos da madeira.

Palavras-chave: Floresta Amazônica; taper; valor ponderado de escores estatísticos.
\end{abstract}

\begin{abstract}
Evaluation of taper functions for diameter estimated of commercial forest species in amazon matogrossense biome. The aim of this work was to adjust, evaluate and select taper functions for estimating diameters along the stem for commercially exploited species in the Amazon Rainforest in Mato Grosso State. The research was conducted in forest management units in areas of the Amazon Rainforest, located in Santa Carmen, Mato Grosso State. To adjust the taper functions, 449 trees were cubed, of the following species: Goupia glabra, Qualea albiflora, Trattinnickia burseraefolia, Erisma uncinatam and Mezilaurus Itauba. The non-segmented models of Schöepfer (1966), Kozak et al. (1969), Hradetzky (1976), and Demaerschalk (1972), adapted for Moura (1994), and segmented model of Max e Burkhart (1976) were fitted. In order to evaluate the accuracy of adjustments we used the determination coefficient, standard error of estimate in percentage and graphical analysis of the waste, weighted value of statistical scores, and auxiliary statistics: standard deviation, standard deviation of differences, sum of squared residuals and relative percent residue. The function of taper Demaerschalk (1972) was the most accurate to estimate the diameter along the stem for forest species of Amazon Matogrossense. Such function allows to describe the shape of the stem as well as the possibility of determining assortments for multiple products of wood.

Keywords: Amazon Rainforest; taper; weighted value of statistical scores.
\end{abstract}

\section{INTRODUÇÃO}

A redução drástica do estoque de madeira nas florestas tropicais, decorrente da expansão da 
agricultura e da exploração, além dos desperdícios ocasionados pelos processos de transformação, demonstram a necessidade de otimização do uso dos recursos madeireiros dentro do conceito de múltiplos produtos, buscando a rentabilidade máxima. Entretanto, nessas florestas, é possível observar a existência da grande variação nas formas dos fustes das árvores, que, geralmente, está relacionada com a diminuição dos diâmetros da base ao topo, denominada de afilamento, a qual, para Silva e Paula Neto (1979) e Ferreira e Silva (2002), é a razão fundamental da variação no volume das árvores.

A descrição do perfil longitudinal dos fustes das árvores é uma operação complexa, uma vez que o tronco não segue o mesmo padrão de forma em toda a sua extensão e, de modo geral, as formas dos fustes são comparadas a diversos sólidos geométricos, denominados de protótipos dendrométricos, com as maiores irregularidades ocorrendo nas porções inferiores dos troncos (FAVALESSA et al., 2012b). Assim, a existência de grande variabilidade na forma do fuste acarreta significativo impacto na determinação do volume explorado nas florestas tropicais do bioma Amazônico, em que, no cálculo da volumetria em inventários florestais, são utilizados, de modo geral, valores padronizados de fatores de formas sem considerar a diferença existente entre as espécies, o que visa basicamente à simplificação dos processos técnicos, porém acarreta a estimativa inadequada do estoque madeireiro.

Entre as principais causas da diferença na forma dos troncos em florestas, pode-se citar o grau de competição, relacionado com o decréscimo no afilamento, e a associação com o comprimento de copa, posição sociológica, idade e espécies em diversos estágios de desenvolvimento (LARSON, 1963; SCOLFORO, 2005; QUEIROZ et al., 2008; MIGUEL et al., 2011; SILVA et al., 2011). Isso tem induzido à pesquisa e ao desenvolvimento de modelos matemáticos aplicados ao manejo de florestas naturais que auxiliem na definição do uso e da transformação da madeira em produto final (CHICHORRO et al., 2003), o que é de fundamental importância para a definição de planos de manejo que visam o uso de múltiplos produtos da floresta.

Atualmente, no setor florestal, são utilizadas técnicas apropriadas para a quantificação e a qualificação dos produtos madeireiros, como as funções de afilamento, as quais correspondem a uma excelente opção para o aprimoramento das técnicas de inventários florestais e quantificação dos sortimentos (CHICHORRO et al., 2003; QUEIROZ et al., 2008; SOUZA et al., 2008; SOUZA et al., 2012; YOSHITANI JÚNIOR et al., 2012), uma vez que permitem descrever matematicamente o perfil longitudinal de um tronco e determinar o volume de madeira por meio da integral dessa função, entre quaisquer pontos ao longo do fuste (AHRENS; HOLBERT, 1981; MENDONÇA et al., 2007), além de permitirem, também, a estimativa de diâmetros mínimos de cada produto e, dessa maneira, otimizarem o uso do fuste (HORLE et al., 2010).

As funções de afilamento podem ser caracterizadas por modelos segmentados e não segmentados. Os não segmentados são constituídos de um único modelo para toda a extensão dos fustes, ao passo que os segmentados constituem-se do uso de submodelos justapostos, cada um representando uma porção do fuste, em que a união destes se dá por meio da imposição de restrições ao modelo geral ajustado para seções inferiores, médias e superiores do fuste (ASSIS et al., 2001; PIRES; CALEGÁRIO, 2007; FAVALESSA et al., 2012b).

As pesquisas que têm como foco analisar o perfil do tronco das árvores são voltadas, principalmente, para os povoamentos florestais implantados com as espécies dos gêneros Eucalyptus e Pinus (QUEIROZ et al., 2006), com destaque a alguns trabalhos desenvolvidos para espécies florestais nativas do bioma Amazônico, tais como Moura (1994) e Leite et al. (2006). Nesse sentido, o objetivo do presente trabalho foi ajustar, avaliar e selecionar funções de afilamento para a estimativa de diâmetros ao longo do fuste de espécies florestais comercialmente exploradas na Amazônia Mato-grossense.

\section{MATERIAL E MÉTODOS}

O estudo foi desenvolvido em unidades de manejo florestal da Floresta Amazônica localizadas no município de Santa Carmem, estado de Mato Grosso, entre as coordenadas geográficas $12^{\circ} 00^{\prime} \mathrm{S}$ e $12^{\circ} 10^{\prime} \mathrm{S}$ de latitude e $54^{\circ} 40^{\prime} \mathrm{W}$ e $54^{\circ} 50^{\prime} \mathrm{W}$ de longitude.

O clima da região é do tipo Aw, segundo a classificação de Köppen, com precipitação pluviométrica de $2.500 \mathrm{~mm}$ a $2.750 \mathrm{~mm}$ e um período curto com precipitação inferior a $60 \mathrm{~mm}$ no mês mais seco. Apresenta temperatura média de $25^{\circ} \mathrm{C}$, com a estação seca coincidindo com o inverno. A vegetação é do tipo Floresta Ombrófila Densa Tropical em estágio clímax de desenvolvimento, com um grande número 
de espécies, muitas de excelentes propriedades e de ótima potencialidade madeireira (BRASIL, 1980).

Foram selecionadas cinco espécies comercialmente exploradas na Floresta Amazônica, segundo a avaliação do Instituto do Homem e Meio Ambiente da Amazônia (IMAZON, 2000): Goupia glabra Aubl. (cupiúba), Qualea albiflora Warm. (cambará), Trattinnickia burseraefolia Willd. (amescla), Erisma uncinatam Warm. (cedrinho) e Mezilaurus itauba (Meissn.) Taub. (itaúba), cujos fins se destinam a marcenaria, laminados, compensados e construção civil.

Foram cubados 449 indivíduos pelo método de Smalian, com o abate das árvores e a medição dos diâmetros, com a casca, a 0,1 $\mathrm{m}$ do solo, a $1,3 \mathrm{~m}$ e a cada $2,0 \mathrm{~m}$ ao longo do fuste até a altura comercial, definida da base ao início da copa, cuja análise estatística descritiva das variáveis encontra-se na tabela 1.

Tabela 1. Análise estatística descritiva do diâmetro a 1,3 $\mathrm{m}$ do solo e da altura comercial para as espécies florestais em estudo da Amazônia Mato-grossense.

Table 1. Descriptive statistical analysis of diameter $1.3 \mathrm{~m}$ and commercial height for forest species in study from Matogrossense Amazon.

\begin{tabular}{lcccccc}
\hline Estatísticas & Mínimo & Média & Máximo & Desvio padrão & Variância & $\begin{array}{c}\text { Coeficiente } \\
\text { de variação (\%) }\end{array}$ \\
\hline Diâmetro a 1,3 m do solo (cm) & 34,70 & 70,60 & 158,28 & 19,85 & 393,96 & 28,12 \\
Altura comercial (m) & 5,30 & 13,59 & 21,75 & 2,81 & 7,87 & 20,66 \\
\hline
\end{tabular}

Foram ajustadas cinco funções de afilamento (Tabela 2) para o agrupamento dos dados das espécies estudadas, sendo avaliados os modelos não segmentados de Schöepfer (1966), Kozak et al. (1969), Hradetzky (1976) e Demaerschalk (1972) adaptado por Moura (1994), além do modelo segmentado de Max e Burkhart (1976). Para o ajuste do modelo proposto por Hradetzky (1976), foram utilizadas as potências 0,$005 ; 0,01 ; 0,02 ; 0,03 ; 0,04 ; 0,05 ; 0,06 ; 0,07 ; 0,08 ; 0,09 ; 0,1 ; 0,2 ; 0,3 ; 0,4 ; 0,5$; 0,$6 ; 0,7 ; 0,8 ; 0,9 ; 1 ; 2 ; 3 ; 4 ; 5 ; 6 ; 7 ; 8 ; 9 ; 10 ; 15$ e 25, com a seleção realizada pelo processo stepwise do programa estatístico R, assim como proposto por Assis et al. (2002), Figueiredo et al. (2006), Mendonça et al. (2007), Pires e Calegário (2007), Souza et al. (2008b) e Miguel et al. (2011). Para a modelagem da função de Max e Burkhart (1976), foi utilizada a ferramenta Solver do Microsoft Excel, com o uso do algoritmo Newton e impondo as restrição aos pontos de ligação dos polinômios $\left(a_{\mathrm{i}}\right)$ de $0,1<\mathrm{a}_{1} \leq 0,3 \mathrm{e}$ $0,3<\mathrm{a}_{2} \leq 0,99$, buscando a convergência do modelo, conforme indicado em trabalhos desenvolvidos por Figueiredo Filho e Schaaf (1996), Assis et al. (2001), Souza et al. (2008b) e Favalessa et al. (2012b).

Tabela 2. Funções de afilamento ajustadas para as espécies florestais em estudo da Amazônia Matogrossense.

Table 2. Taper functions fitted for forest species in study from Matogrossense Amazon.

\begin{tabular}{ll}
\hline Modelo & \multicolumn{1}{c}{ Ajuste } \\
\hline Schöepfer (1966) & $\frac{d_{i}}{D}=\beta_{0}+\beta_{1}\left(\frac{h_{i}}{H}\right)+\beta_{2}\left(\frac{h_{i}}{H}\right)^{2}+\beta_{3}\left(\frac{h_{i}}{H}\right)^{3}+\beta_{4}\left(\frac{h_{i}}{H}\right)^{4}+\beta_{5}\left(\frac{h_{i}}{H}\right)^{5}$ \\
Kozak et al. (1969) & $\left(\frac{d_{i}}{D}\right)^{2}=\beta_{0}+\beta_{1}\left(\frac{h_{i}}{H}\right)+\beta_{2}\left(\frac{h_{i}}{H}\right)^{2}$ \\
Hradetzky (1976) & $\frac{d_{i}}{D}=\beta_{0}+\beta_{1}\left(\frac{h_{i}}{H}\right)^{p_{1}}+\beta_{2}\left(\frac{h_{i}}{H}\right)^{p_{2}}+\ldots+\beta_{n}\left(\frac{h_{i}}{H}\right)^{p_{n}}$ \\
$\begin{array}{l}\text { Demaerschalk (1972) } \\
\text { adaptado por Moura (1994) }\end{array}$ & $\frac{d_{i}}{D}=\beta_{0}+\beta_{1}\left(\frac{1}{h_{i}+1,3}\right)^{\beta_{2}} H^{\beta_{3}}$ \\
Max e Burkhart (1976) & $\frac{d_{i}}{D}=\left[\beta_{1}(X-1)+\beta_{2}\left(X^{2}-1\right)+\beta_{3}\left(a_{1}-X\right)^{2} I_{1}+\beta_{4}\left(a_{2}-X\right)^{2} I_{2}\right]^{0,5}$ \\
\hline
\end{tabular}

$\beta_{\mathrm{i}}$ : coeficientes de regressão; $\mathrm{d}_{\mathrm{i}}$ : diâmetro mensurado a uma altura $\mathrm{h}_{\mathrm{i}}(\mathrm{cm}) ; \mathrm{D}$ : diâmetro a $1,3 \mathrm{~m}$ do solo $(\mathrm{cm}) ; \mathrm{H}$ : altura comercial $(\mathrm{m}) ; \mathrm{h}_{\mathrm{i}}$ : altura a uma seção i (m); $\mathrm{p}_{1}, \mathrm{p}_{2}$ e $\mathrm{p}_{\mathrm{n}}$ : potências; $\mathrm{a}_{1}$ e $\mathrm{a}_{2}$ : pontos de ligação dos polinômios; $\mathrm{X}: \mathrm{h}_{\mathrm{i}} / \mathrm{H} ; \mathrm{I}_{\mathrm{i}}: 1$ se $\mathrm{X}<\mathrm{a}_{\mathrm{i}}$; e $\mathrm{I}_{\mathrm{i}}$ : 0 se $\mathrm{X}>\mathrm{a}_{\mathrm{i}}$.

Avaliação dos ajustes

Para avaliar a acuracidade das funções de afilamento, foram utilizadas as estatísticas erro padrão 
de estimativa em porcentagem $\left(S_{\mathrm{yx}} \%\right)$ e coeficiente de determinação ajustado $\left(\mathrm{R}_{\text {aj. }}{ }^{\mathrm{j}}\right)$, as quais foram ordenadas de acordo com a eficiência, sendo atribuído peso 1 para a equação mais eficiente e pesos crescentes para as demais, conforme a metodologia do valor ponderado dos escores estatísticos aplicada por Thiersch (1997) e Hess e Schneider (2010). Nesse processo, o valor ponderado de uma equação foi obtido pela multiplicação do número de vezes quantificado para cada colocação pelo seu respectivo peso, sendo selecionada a equação que proporcionou o menor valor ponderado, expresso por:

$$
V P=\sum_{i=1}^{n} N r_{i} \cdot P_{i}
$$

Em que: $\mathrm{VP}=$ valor ponderado da equação;

$\mathrm{P}_{\mathrm{i}}=$ peso da iésima colocação;

$\mathrm{Nr}_{\mathrm{i}}=$ número de registros que obtiveram a iésima colocação.

Além disso, procedeu-se à análise gráfica dos resíduos e à comparação dos perfis médios observados aos estimados. Também foram calculadas as estatísticas desvio, desvio padrão das diferenças, somatório dos quadrados dos resíduos relativos e resíduos percentuais (Tabela 3), utilizadas por Figueiredo Filho et al. (1996), Scolforo et al. (1998), Assis et al. (2002) e Favalessa et al. (2012), as quais permitiram a avaliação do desempenho das funções nas estimativas dos diâmetros ao longo do fuste.

Tabela 3. Estatísticas para a avaliação das estimativas dos diâmetros pelas funções de afilamento ajustadas para as espécies florestais da Amazônia Mato-grossense.

Table 3. Statistics for the evaluation of diameters estimated by taper functions fitted for forest species from Matogrossense Amazon.

\begin{tabular}{lc}
\hline Estatística & Formulação \\
\hline Desvio & $\left(\Sigma\right.$ Diff $\left._{i}\right) / n$ \\
Desvio padrão das diferenças & $\Sigma\left(\text { Diff }_{i} / d_{i}\right)^{2} /(n-1)$ \\
Somatório dos quadrados dos resíduos relativos & $\Sigma\left(\text { Diff }_{i} / d_{i}\right)^{2}$ \\
Resíduos percentuais & {$\left[\Sigma\left(\right.\right.$ Diff $\left.\left._{i} / d_{i}\right) .100\right] / n$} \\
\hline
\end{tabular}

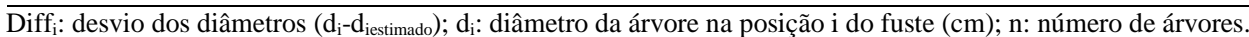

\section{RESULTADOS E DISCUSSÃO}

$\mathrm{Na}$ tabela 4 estão apresentados os coeficientes de regressão, os erros padrões de estimativa em porcentagem $\left(\mathrm{S}_{\mathrm{yx}} \%\right)$ e os coeficientes de determinação ajustados $\left(\mathrm{R}_{\text {aj. }}^{2}\right)$ das funções de afilamento ajustadas para as espécies florestais da Amazônia Mato-grossense.

Tabela 4. Coeficientes e estatísticas de ajuste e precisão das funções de afilamento ajustadas para as espécies florestais da Amazônia Mato-grossense.

Table 4. Coefficients and statistics of adjustment and precision of taper functions adjusted for forest species from Matogrossense Amazon.

\begin{tabular}{|c|c|c|c|c|c|c|c|c|c|c|}
\hline \multirow{2}{*}{ Função } & \multicolumn{5}{|c|}{ Coeficientes estimados } & \multicolumn{5}{|c|}{ Estatísticas } \\
\hline & $\mathbf{a}_{1}$ & $\mathbf{a}_{2}$ & $\boldsymbol{\beta}_{0}$ & $\beta_{1}$ & $\boldsymbol{\beta}_{2}$ & $\boldsymbol{\beta}_{3}$ & $\boldsymbol{\beta}_{4}$ & $\beta_{5}$ & $\mathbf{S}_{\mathrm{yx}} \%$ & $\mathbf{R}_{\text {aj. }}^{2}$ \\
\hline Schöepfer (1966) & & & 1,210 & $-2,945$ & 10,940 & $-20,46$ & 17,92 & $-5,901$ & 9,49 & 0,902 \\
\hline Kozak et al. (1969) & & & 1,211 & $-1,475$ & 0,896 & & & & 10,04 & 0,889 \\
\hline Hradetzky (1976) & & & $\begin{array}{c}21,59 \\
6\end{array}$ & $-20,826$ & & & & & 9,49 & 0,901 \\
\hline $\begin{array}{l}\text { Demaerschalk } \\
\text { (1972) adaptado }\end{array}$ & & & 1,783 & 0,925 & 0,177 & $-0,023$ & & & 8,48 & 0,922 \\
\hline $\begin{array}{l}\text { Max e Burkhart } \\
(1976)\end{array}$ & 0,191 & 0,989 & & 11272,5 & $-5693,7$ & 13,078 & 5694 & & 8,95 & 0,913 \\
\hline
\end{tabular}

Os modelos apresentaram resultados satisfatórios, com $\mathrm{S}_{\mathrm{yx}} \%$ entre $8,48 \%$ e $10,04 \%$ e $\mathrm{R}_{\text {aj. }}^{2}$ de 0,889 a 0,922 , sendo o melhor ajuste verificado pelo modelo de Demaerschalk (1972) adaptado, semelhante ao constatado nos trabalhos de Moura (1994) com espécies nativas da Floresta Amazônica, 
seguido pelas funções de Max e Burkhart (1976), Hradetzky (1976) e Schöepfer (1966), enquanto o modelo de Kozak et al. (1969) mostrou o pior ajuste.

Visando obter uma definição clara da equação de afilamento mais adequada para estimar os diâmetros ao longo do tronco, foi aplicado o método do valor ponderado dos escores estatísticos (VP). Os resultados são apresentados na tabela 5, em que foi observado o menor VP para o modelo de Demaerschalk (1972) adaptado, seguido pelos de Max e Burkhart (1976) e Schöepfer (1966), ao passo que as funções de Hradetzky (1976) e Kozak et al. (1969) forneceram os maiores somatórios dos escores estatísticos, comprovando a eficácia da metodologia na seleção de funções de afilamento adequadas às espécies florestais em estudo da Amazônia Mato-grossense.

Tabela 5. Valor ponderado dos escores das estatísticas das funções de afilamento ajustadas para as espécies florestais em estudo da Amazônia Mato-grossense.

Table 5. Weighted value and scores of statistics of taper functions adjusted for forest species in study from Matogrossense Amazon.

\begin{tabular}{lccc}
\hline \multirow{2}{*}{ Função } & \multicolumn{3}{c}{ Escores das estatísticas } \\
\cline { 2 - 4 } & $\mathbf{S}_{\mathbf{y x}} \%$ & $\mathbf{R}_{\text {aj. }}^{\mathbf{2}}$ & Valor ponderado \\
\hline Schöepfer (1966) & 3 & 3 & 6 \\
Kozak et al. (1969) & 4 & 5 & 9 \\
Hradetzky (1976) & 3 & 4 & 7 \\
Demaerschalk (1972) adaptado & 1 & 1 & 2 \\
Max e Burkhart (1976) & 2 & 2 & 4 \\
\hline
\end{tabular}

A avaliação geral dos modelos foi realizada considerando não somente as estatísticas $R_{\text {aj. }}^{2}$ e $\mathrm{S}_{\mathrm{yx}} \%$, mas também a análise gráfica dos resíduos (Figura 1), o que possibilitou verificar a magnitude do afastamento dos valores dos diâmetros estimados, em relação aos observados, em todo o fuste. Assim, foi verificado que os modelos ajustados forneceram uma boa dispersão residual, com proporcionalidade entre valores subestimados e superestimados, principalmente para o modelo de Demaerschalk (1972) adaptado por Moura (1994).

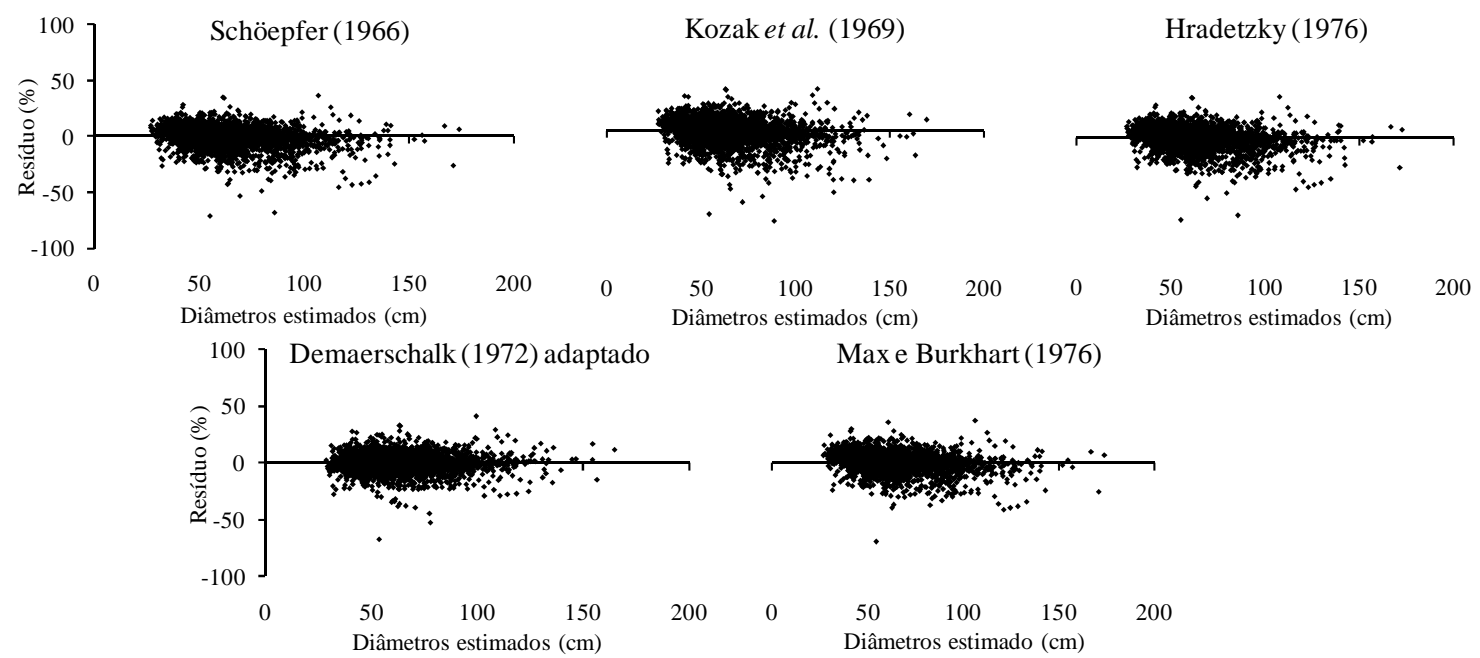

Figura 1. Distribuição gráfica dos resíduos (\%) para as estimativas dos diâmetros, por meio das funções de afilamento, para as espécies florestais da Amazônia Mato-grossense.

Figure 1. Graphic distribution of residues (\%) for estimates of the diameters by taper functions for forest species from Matogrossense Amazon.

As tendências nas estimativas dos diâmetros também foram analisadas pela comparação dos perfis médios observados aos estimados (Figura 2). Assim, foram observadas superestimativas dos diâmetros nas porções superiores do tronco, possivelmente devido à dificuldade de relacionar a ponta do 
fuste das folhosas aos protótipos dendrométricos, contradizendo a teoria do afilamento constante do fuste aplicada às coníferas, como observado por Figueiredo et al. (1996) e Favalessa et al. (2012b). No entanto, o perfil médio estimado pela função de Demaerschalk (1972) adaptado por Moura (1994) apresentou maior semelhança ao perfil médio observado, enquanto o modelo de Kozak et al. (1969) proporcionou os maiores afastamentos das estimativas dos diâmetros, com subestimativas até, aproximadamente, $6 \mathrm{~m}$, seguido por superestimativas nas seções subsequentes a $13 \mathrm{~m}$ do tronco.
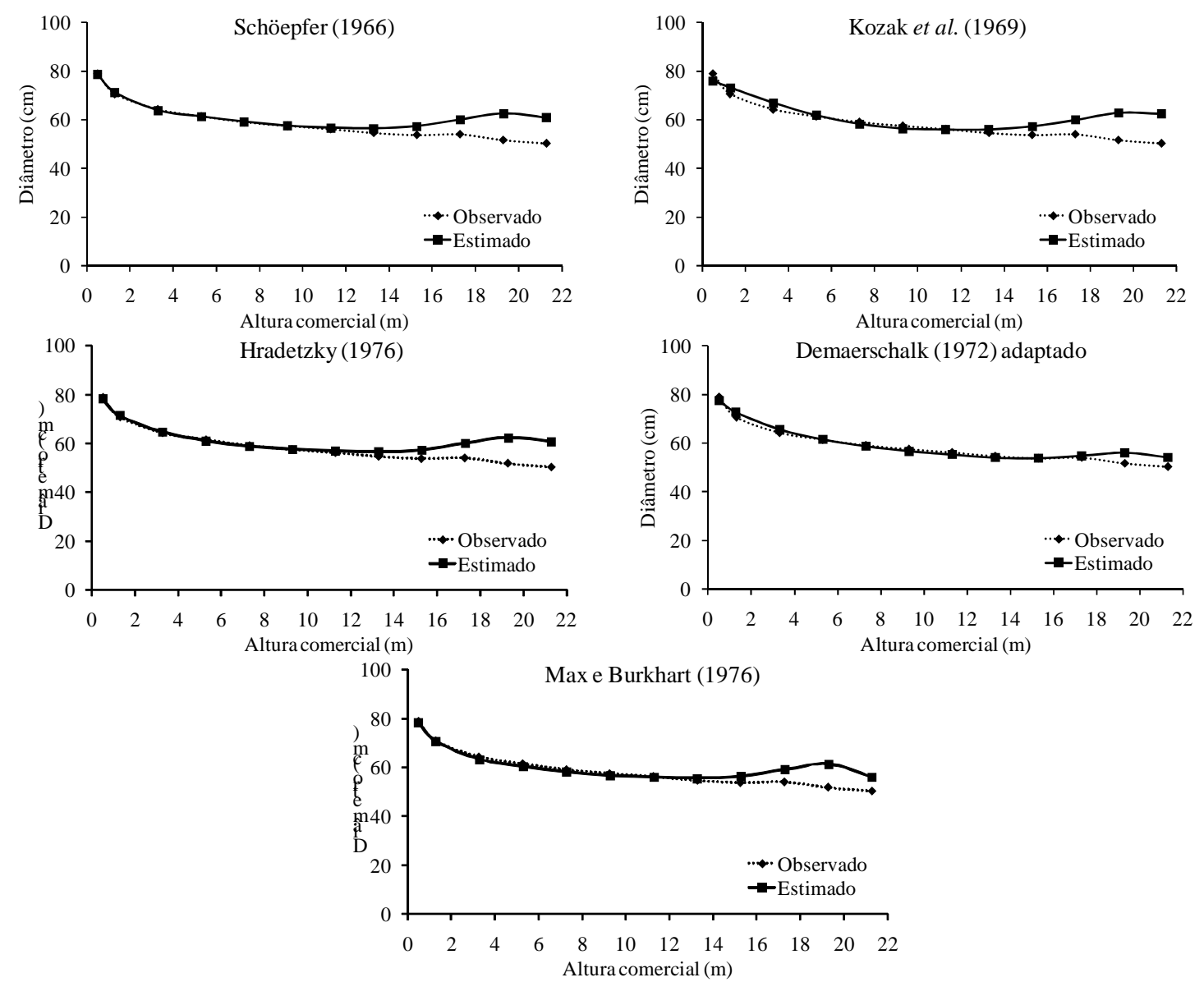

Figura 2. Perfis médios observados e estimados pelas funções de afilamento para as espécies florestais em estudo da Amazônia Mato-grossense.

Figure 2. Observed and estimated profiles by taper functions for forest species in study from Matogrossense Amazon.

A avaliação geral feita pelas estatísticas $\mathrm{R}_{\text {aj. }}^{2}$ e $\mathrm{S}_{\mathrm{yx}} \%$ retratam valores médios e não fornecem indicativos do comportamento ao longo do fuste, de modo que foi realizada a análise da acuracidade dos modelos para as estimativas dos diâmetros ao longo do tronco, por meio das estatísticas de desvio, soma de quadrados dos resíduos relativos, desvio padrão das diferenças e resíduo percentual, com os resultados da seleção das funções de afilamento apresentados na tabela 6 .

As análises estatísticas demonstraram que o modelo de Demaerschalk (1972) adaptado estimou, com maior acuracidade, os diâmetros em um maior número de alturas de medição, enquanto a função de Max e Burkhart (1976) e Schöepfer (1966) foram as mais adequadas para descrever a base do tronco, e os modelos de Kozak et al. (1969) e Hradetzky (1976) foram pouco acurados.

De modo geral, as funções de afilamento testadas forneceram os melhores ajustes para o conjunto das espécies estudadas, sendo superiores aos resultados obtidos por Müller et al. (2005) ao trabalhar com a altura total de Eucalyptus grandis no município de Guaíba, estado do Rio Grande do Sul, 
por Leite et al. (2006) com a altura comercial de Virola surinamensis no município de Afuá, estado do Pará, por Queiroz (2006), Queiroz et al. (2006) e Queiroz et al. (2008) com a altura total de Mimosa scabrella na Região Metropolitana de Curitiba, Paraná, e por Pires e Calegário (2007) com a altura até o limite de 5,0 cm de diâmetro de Eucalyptus sp. em povoamentos nos estados da Bahia e Espírito Santo. Os resultados obtidos neste trabalho para os ajustes das funções de afilamento foram semelhantes aos ajustes obtidos por Moura (1994), ao trabalhar com a altura comercial de diversas espécies nativas da Floresta Amazônica no estado do Amazonas, e por Chichorro et al. (2003), com o modelo de Demaerschalk, para a quantificação de multiprodutos da madeira até a altura comercial de espécies da Floresta Atlântica no município de Conceição da Barra, Espírito Santo.

Tabela 6. Classificação das funções de afilamento para as estimativas de diâmetros ao longo do fuste, para as espécies florestais em estudo da Amazônia Mato-grossense.

Table 6. Classification of taper functions for estimates of diameters, along the stem, for forest species in study from Matogrossense Amazon.

\begin{tabular}{lc}
\hline Altura (m) & Função de afilamento selecionada \\
\hline 0,5 & Max e Burkhart (1976) \\
1,3 & Max e Burkhart (1976) e Schöepfer (1966) \\
3,3 & Max e Burkhart (1976), Schöepfer (1966) e Demaerschalk (1972) adaptado \\
5,3 & Max e Burkhart (1976) \\
7,3 & Demaerschalk (1972) adaptado \\
9,3 & Demaerschalk (1972) adaptado \\
11,3 & Kozak et al. (1969) \\
13,3 & Demaerschalk (1972) adaptado \\
15,3 & Demaerschalk (1972) adaptado \\
17,3 & Demaerschalk (1972) adaptado \\
19,3 & Demaerschalk (1972) adaptado \\
\hline
\end{tabular}

\section{CONCLUSÕES}

- A função de afilamento de Demaerschalk (1972) adaptado por Moura (1994) é a mais acurada para estimar os diâmetros ao longo do fuste das espécies Goupia glabra, Qualea albiflora, Trattinnickia burseraefolia, Erisma uncinatam e Mezilaurus itauba do bioma Amazônico no estado de Mato Grosso, o que permite descrever a forma dos troncos e a possibilidade da determinação de sortimentos para múltiplos produtos da madeira.

- O método do valor ponderado dos escores estatísticos é uma ferramenta eficaz para o auxílio na seleção de funções de afilamento adequadas, enquanto a avaliação das estatísticas de desvio, soma de quadrados dos resíduos relativos, desvio padrão das diferenças e resíduo percentual (RP) permitem determinar a acurácia das funções de afilamento para as estimativas dos diâmetros ao longo do fuste das espécies florestais da Amazônia Mato-grossense.

- As funções de afilamento demonstram maior dificuldade para estimar os diâmetros das seções superiores do tronco, possivelmente devido à baixa relação da ponta do fuste das espécies florestais da Floresta Amazônica aos protótipos dendrométricos.

\section{REFERÊNCIAS}

AHRENS, S.; HOLBERT, D. Uma função para forma de tronco e volume de Pinus taeda L. Boletim de Pesquisa Florestal, v. 3, p. 37 - 68, 1981.

ASSIS, A. L. de; SCOLFORO, J. R. S.; MELLO, J. M. de; ACERBI JÚNIOR, F. W.; OLIVEIRA, A. D. de. Comparação de modelos polinomiais segmentados e não segmentados na estimativa de diâmetros e volumes ao longo do fuste de Pinus taeda. Cerne, v. 7, n. 1, p. 20 - 40, 2001.

ASSIS, A. L. de; SCOLFORO, J. R. S.; MELLO, J. M. de; OLIVEIRA, A. D. de. Avaliação de modelos polinomiais não segmentados na estimativa de diâmetros e volumes comerciais de Pinus taeda. Ciência 
Florestal, v. 12, n. 1, p. 89 - 107, 2002.

BRASIL. Folha SD. 21 - Juruena: geologia, geomorfologia, pedologia, vegetação e uso potencial da terra. Rio de Janeiro: Ministério das Minas e Energia, 1980. 464 p.

CHICHORRO, J. F.; RESENDE, J. L. P.; LEITE, H. G. Equações de volume e de taper para quantificar multiprodutos da madeira em Floresta Atlântica. Árvore, v. 27, n. 6, p. 799 - 809. 2003.

DEMAERSCHALK, J. P. Converting volume equations to compatible taper equations. Forest Science, v. 18, n. 3, p. 241 - 245, 1972.

FAVALESSA, C. M.; UBIALLI, J. A.; CALDEIRA, S. F.; DRESCHER, R. Equações de sortimentos para Tectona grandis na região centro-sul de Mato Grosso Pesquisa Florestal Brasileira, v. 32, n. 72, p. 389 - 399, 2012.

. Funções de afilamento não segmentadas e segmentadas para Tectona grandis na região centro-sul mato-grossense. Pesquisa Florestal Brasileira, v. 32, n. 72, p. 373 - 387, 2012.

FERREIRA, C. A.; SILVA, H. D. da. Afilamento e forma do tronco de árvores de Eucalyptus grandis e Eucalyptus saligna e suas variações com a adubação. Boletim de Pesquisa Florestal, n. 44, p. 87 - 106, 2002.

FIGUEIREDO, E. O.; SCOLFORO, J. R. S.; OLIVEIRA, A. D. de. Seleção de modelos polinomiais para representar o perfil e volume do fuste de Tectona grandis L. f. Acta Amazonica, v. 36, n. 4, p. 465 - 482, 2006.

FIGUEIREDO FILHO, A.; BORDERS, B. E.; HITCH, K. L. Taper equations for Pinus taeda plantations in southern Brazil. Forest Ecology and Management, v. 83, n. 1/2, p. 36 - 46, 1996.

FIGUEIREDO FILHO, A.; SCHAAF, L. B. Comparison between predicted volumes estimated by taper equations and true volumes obtained by the water displacement technique (xylometer). Canadian Journal of Forest Research, v. 29, p. 451 - 461, 1999.

HESS, A. F.; SCHNEIDER, P. R. Crescimento em volume de Araucaria angustifolia (Bertol.) Kuntze em três regiões do Rio Grande do Sul, Brasil. Ciência Florestal, v. 20, n. 1, p. 107 - 122, 2010.

HORLE, D. C.; MENDONÇA, A. R. de; CARVALHO, S. de P. C.; CALEGÁRIO, N. Modelagem não linear do perfil longitudinal de fustes de Pinus oocarpa. Cerne, v. 16, n. 2, p. 177 - 184, 2010.

HRADETZKY, J. Analysis und interpretation statistisher abränger keiten. Baden: Württemberg Mitteilungen der FVA, 1976. $146 \mathrm{p}$.

INSTITUTO DO HOMEM E MEIO AMBIENTE DA AMAZÔNIA (IMAZON). Rendimento no processamento de madeiras no estado do Pará. Série Amazônia, n. 18, 2000.

KOZAK, A.; MUNRO, D. P.; SMITH, J. H. G. Taper functions and their application in forest inventory. Forest Chronicle, v. 45, n. 4, p. 278 - 283, 1969.

LARSON, P. R. Stern from development of forest tree. Forest Science Monograph, p. 1 - 41, 1963.

LEITE, H. G.; GAMA, J. R. V.; CRUZ, J. P., SOUZA, A. L. Função de afilamento para Virola surinamensis (Roll.) Warb. Árvore, v. 30, n. 1, p. 99 - 106. 2006.

MAX, T. A.; BURKHART, H. E. Segmented polynomial regression applied to taper equations. Forest Science, v. 22, n. 3, p. 283 - 289, 1976.

MENDONÇA, A. R. de; SILVA, G. F. da; OLIVEIRA, J. T. da S.; NOGUEIRA, G. S.; ASSIS, A. L. de. Avaliação de funções de afilamento visando a otimização de fustes de Eucalyptus sp. para multiprodutos. Cerne, v. 13, n. 1, p. 71 - 82, 2007.

MIGUEL, E. P.; MACHADO, S. do A.; FIGUEIREDO FILHO, A.; ARCE, J. E. Modelos polinomiais para representar o perfil e o volume do fuste de Eucalyptus urophylla na região norte do estado de Goiás. Floresta, v. 41, n. 2, p. 355 - 368, 2011. 
MOURA, J. B. Estudo da forma do fuste e comparação de métodos de estimativa volumétrica de espécies florestais da Amazônia brasileira. 114 f. Dissertação (Mestrado em Engenharia Florestal) Universidade Federal do Paraná, Curitiba, 1994.

MÜLLER, I.; FINGER, C. A.; SCHNEIDER, P. R. Forma de tronco e sortimentos de madeira de Eucalyptus grandis Hill ex Maiden, na região sudeste do estado do Rio Grande do Sul. Ciência Florestal, v. 15, n. 3, p. 293 - 305. 2005.

PIRES, L. M.; CALEGÁRIO, N. Ajuste de modelos estocásticos lineares e não lineares para a descrição do perfil longitudinal de árvores. Árvore, v. 31, n. 5, p. 845 - 852, 2007.

QUEIROZ, D.; MACHADO, S. do A.; FIGUEIREDO FILHO, A.; ARCE, J. E.; KOEHLER, H. S. Avaliação e validação de funções de afilamento para Mimosa scabrella Bentham em povoamentos da Região Metropolitana de Curitiba/PR. Floresta, v. 36, n. 2, p. 183 - 199, 2006.

Identidade de modelos em funções de afilamento para Mimosa scabrella Bentham em povoamentos nativos da região metropolitana de Curitiba/PR. Floresta, v. 38, n. 2, p. 339 - 349, 2008.

SCHOEPFER, W. Automatisierung des Massen, Sorten und. Wertberechnung stehender Waldbestände. Schriftenreihe. Bad. Wurtt-Forstl., v. 21, 1966.

SCOLFORO, J. R. S. Biometria Florestal: modelos de regressão linear e não linear; modelos para relação hipsométrica, volume, afilamento e peso de matéria seca. Lavras: UFLA/FAEPE, 2005. 352 p.

SCOLFORO, J. R. S.; RIOS, M. S.; OLIVEIRA, A. D. de; MELlO, J. M. de; MAESTRI, R. Acuracidade de equações de afilamento para representar o perfil do fuste de Pinus elliottii. Cerne, v. 4, n. 1 , p. 100 - 122, 1998.

SILVA, F. da; DALLA CORTE, A. P.; SANQUETTA, C. R. Equações de afilamento para descrever o volume total do fuste de Pinus caribaea var. hondurensis na região do Triângulo Mineiro. Scientia Forestalis, v. 39, n. 91, p. 367 - 376, 2011.

SILVA, J. A. A.; PAUlA NETO, F. Princípios de dendrometria. Universidade Federal Rural de Pernambuco. 1979. 198 p.

SOUZA, C. A. M. de; CHASSOT, T.; FINGER, C. A. G.; SCHNEIDER, P. R.; FLEIG, F. D. Modelos de afilamento para o sortimento do fuste de Pinus taeda L. Ciência Rural, v. 38, n. 9, p. 2506 - 2511, 2008.

SOUZA, C. A. M. de; SILVA, G. F. da; XAVIER, A. C.; MENDONÇA, A. R. de; ALMEIDA, A. Q. de. Avaliação de modelos de afilamento não segmentados na estimação da altura e volume comercial de Eucalyptus sp. Ciência Florestal, v. 18, n. 3, p. 387 - 399, 2008.

SOUZA, C. A. M. de; FINGER, C. A. G.; SCHNEIDER, P. R.; SILVA, G. F. da; THOMAS, C. Eficiência de um modelo de afilamento ajustado sem e com estratificação por classe de quociente de forma para formação dos sortimentos de Pinus taeda L. Ciência Florestal, v. 22, n. 1, p. 125 - 135, 2012.

THIERSCH, A. Eficiência das distribuições diamétricas para prognose da produção de Eucalyptus camaldulensis. 155 f. Dissertação (Mestrado em Ciência Florestal) - Universidade Federal de Lavras, Lavras, 1997.

YOSHITANI JÚNIOR, M.; NAKAJIMA, N. Y.; ARCE, J. A.; MACHADO, S. A do.; DRUSZCZ, J. P.; HOSOKAWA, R. T.; MELlO, A. A. Funções de afilamento para plantios desbastados de Pinus taeda. Floresta, v. 42, n. 1, p. 169 - 176, 2012. 
